\title{
25 Research Square \\ Spatiotemporal Cluster Patterns of Hand, Foot, and Mouth Disease at the Province level in Mainland China, 2009-2018
}

\section{Yuanzhe Wu}

University of New South Wales

Tingwei Wang

Central South University

Mingyi Zhao

Central South University

Shumin Dong

FuJian Medical University

Shiwen Wang

Central South University

Jingcheng Shi ( $\nabla$ jingzhengs@126.com )

Central South University

\section{Research Article}

Keywords: Hand, foot, and mouth disease, Epidemiology, Spatial autocorrelation, Spatiotemporal cluster

Posted Date: January 14th, 2022

DOI: https://doi.org/10.21203/rs.3.rs-1254533/v1

License: (1) This work is licensed under a Creative Commons Attribution 4.0 International License.

Read Full License 


\section{Abstract}

\section{Background}

Although three monovalent EV-A71 vaccines have been launched in mainland China since 2016, hand, foot, and mouth disease (HFMD) still causes a considerable disease burden in China. Vaccines' use may change the epidemiological characters of HFMD. This study aims to analyze the spatiotemporal cluster of HFMD at the province level in mainland China from 2009 to 2018 and compare the difference before and after the vaccines were launched.

Methods

All HFMD cases' data from January 2009 to December 2018 were obtained from the public health science data center given by the Chinese Center for Diseases Control and Prevention. Spatial autocorrelation analysis and space-time scan statistics analysis were used to explore the spatiotemporal distribution pattern of this disease at the provincial level in mainland China.

Results

The median annual incidence of HFMD was 143.22 per 100,000 (ranging from 87.01 to 205.06) in mainland China from 2009 to 2018. Two peaks of infections were observed per year. Children 5 years and under were the main morbid population. The global autocorrelation analysis showed that the spatial distribution of HFMD was presented a significant clustering pattern in each year $(P<0.001)$, and the local autocorrelation analysis indicated that the high incidence areas were clustered in the southern and southeastern coastal provinces. The distribution of HFMD cases was clustered in time and space. The range of cluster time was between April and October. The most likely cluster appeared in the southern coastal provinces (Guangxi, Guangdong, Hainan) from 2010 to 2017 and in the southeastern coastal provinces (Shanghai, Jiangsu, Zhejiang) in 2018.

Conclusion

Changes in the spatiotemporal cluster of HFMD after the launch of EV-A71 vaccines were observed at the province level in mainland China in 2018. It is necessary to advance the EV-A71 vaccination plan, analyze the spatial-temporal distribution characteristics of different enterovirus pathogens of HFMD, and promote HFMD multivalent vaccines.

\section{Introduction}

Hand, foot, and mouth disease (HFMD) is a common infectious disease in childhood caused by various enteroviruses. The most commonly reported pathogens are Enterovirus A71 (EV-A71) and Coxsackievirus A16 (CV-A16)[1]. HFMD can be transmitted through close contact with virus-contaminated objects or contaminated respiratory droplets, water, and food[2]. Symptoms of HFMD typically include flu-like infections, skin eruptions on hands and feet, and oral herpes[3, 4]. Most patients show mild and self- 
limiting symptoms, while few patients may occur complications such as myocarditis, pulmonary edema, and aseptic meningitis. Some severe cases will develop rapidly and eventually death $[5,6]$. HFMD has a severe impact on children's health under five years $[1,5]$.

In the past two decades, some Asian countries frequently reported outbreaks of HFMD, which has become a significant public health problem across the Asia-Pacific region[7-11]. HFMD is prevalent throughout China as a perennial disease[2]. By 2018, the Chinese Center for Disease Control and Prevention (Chinese CDC) had reported 20.5 million cases of HFMD, including 3,667 deaths. HFMD has caused an enormous burden of disease in China[12]. In May 2008, HFMD was classified as a category C notifiable infection in China. Medical institutions have been required to report it through the Notifiable Infectious Disease Reporting System (NIDRIS) within 24 hours[13].

Affected by factors such as average temperature, average relative humidity, monthly precipitation, population density, environmental sanitation, etc., the spatial distribution of the HFMD incidence shows differences[14, 15]. From the perspective of temporal dimension, the peak of HFMD occurs in summer in temperate regions, but in the tropical areas, HFMD can occur at any time of the year[16]. Some researchers have found evidence of spatial distribution patterns and spatiotemporal clusters of HFMD [14, 17-20]. Analyzing the spatiotemporal cluster patterns of infections can contribute to identifying high-risk areas and populations, exploring disease risk factors, formulating effective measures to prevent and control the spread of diseases, and allocating public health resources reasonably[14, 17 , $20,21]$. Because the risk of HFMD varies with space and time, it is imperative to determine the spatiotemporal clusters of HFMD in China [22].

Some studies have shown that latitude will affect the incidence of HFMD to a certain extent, and the incidence of HFMD is higher in low latitudes such as tropical and temperate regions[23]. Mainland China spans multiple temperature zones. Natural and social factors vary greatly between the south and north, coastal and inland areas, and the prevalence patterns of HFMD are different in different regions[23, 24]. The incidence of HFMD in southern China is higher than that in the north, and there are two peaks (summer and autumn) of HFMD each year in south China, while there is only one peak(summer) each year in northern China[1]. From 2008 to 2012, China had reported 7.2 million cases of HFMD, of which were 2457 fatal[1]. Several studies had reported that HFMD spread in the complex space-time domain, and the space-time cluster was detected in mainland China from 2008 to 2012[21, 25]. Since 2016, three inactive monovalent EV-A71 vaccines have been launched in China, which may change the epidemiological characters of HFMD[26]. Since then, researches on the spatial and temporal distribution pattern of HFMD has mainly been concentrated in several provinces and cities, such as Shandong[27], Xinjiang[24], Shaanxi[28]. However, few studies have been conducted in the whole of China. Determining the spatiotemporal cluster variation of HFMD after the vaccine was launched will help define new highrisk areas and periods and provide a basis for relevant departments to implement disease control and prevention policies in critical areas. 
Therefore, this study was aimed to use the surveillance data of HFMD to analyze the spatiotemporal cluster of HFMD at the province level in mainland China from 2009 to 2018 and compare the difference before and after the vaccines were launched.

\section{Methods}

\section{Data Resources and Study Area}

All HFMD cases' data were obtained from the public health science data center given by the Chinese Center for Diseases Control and Prevention (Chinese CDC)[29]. All HFMD cases were diagnosed according to the unified diagnostic criteria issued by the Ministry of Health of China and reported to the infectious disease surveillance system[2]. This HFMD cases database collected all the data reported since the direct online reporting from 2008. The main content included the number of cases and death, incidence and mortality by region, age group, and gender[29]. The incidence of HFMD was calculated as the ratio of the number of cases to the number of permanent residents during the study period. The population data from 2009 to 2015 were collected from Chinese CDC. Due to the lack of data from 2016 to 2018, the remaining data were collected from the National Bureau of Statistics of China(NBSC)[30].

Because this HFMD cases database did not contain data on HFMD cases in Taiwan, Hong Kong, and Macau, the actual study area was mainland China, which includes 22 provinces, 4 municipalities, and 5 autonomous regions, a total of 31 provincial-level administrative regions (excluding Hong Kong, Macau and Taiwan). The geographic data were downloaded from the National Geomatics Center of China(NGCC)[31].

A total of 20048244 cases of HFMD were included in this study, which was reported by 31 provincial administrative regions in Mainland China from January 2009 to December 2018. Microsoft Excel (version 2013, Microsoft Corp, Redmond,WA, USA) was used to build the database, and the incidence of each local administrative region each year was represented in different colors on the map by Tableau (version 2021.3.3, TABLEAU, Seattle, WA, USA).

\section{Spatial autocorrelation analysis}

\section{Global spatial autocorrelation analysis}

The global spatial autocorrelation analysis started from the macro-level of the whole country, compared the mean value of the attribute in the general area and the attribute value on each spatial unit to obtain the average degree of association between the incidence of HFMD in various provinces across the country. That was, to determine whether the incidence of HFMD was clustered nationwide. Global Moran's I is a commonly used global correlation index ranging from -1 to 1[32]. I> 0 indicates a positive spatial correlation at the given significance level. The larger the value, the more pronounced the spatial correlation, high observations accumulate in space with high observations, and low observations accumulate spatially. $I<0$ indicates a negative spatial correlation. The smaller the value, the more 
significant the spatial difference, the tendency of high observations to cluster with low observations. If I $=0$, the observations are randomly distributed in space[33].

\section{Local spatial autocorrelation analysis}

Local indicator of spatial association (LISA) is the analysis index of local spatial autocorrelation analysis[34]. In this study, it was used to measure the degree of spatial dependence between the HFMD incidence of a province and the HFMD incidence of its neighboring provinces and identify its spatial cluster pattern in a local space. There are four spatial correlation patterns: High-High cluster, Low-Low cluster, High-Low cluster, and Low-High cluster. High-High cluster and Low-Low cluster indicate that the observations have a strong positive spatial correlation. The two patterns respectively indicate that the high-incidence areas are adjacent to the high-incidence areas, and the low-incidence areas are adjacent to the low-incidence areas. The High-Low cluster and Low-High cluster signify that the observations have a strong negative spatial correlation. The two patterns mean that high-incidence areas are adjacent to lowincidence areas.

GeoDa (version 1.16.016, GitHub, San Francisco, CA, USA) was used for spatial autocorrelation analysis, and the results of local spatial autocorrelation were displayed on the map with Tableau (version 2021.3.3, TABLEAU, Seattle, WA, USA)

\section{Space-time scan statistics analysis}

The space-time scan statistics analysis was proposed by Kulldorff, which can determine the space-time cluster[35]. The principle is to establish a scanning window that moves in space, the radius of the window is constantly changing according to the population of the study area, and an infinite number of circular windows with different radius are generated to detect possible spatial agglomeration areas. Calculate the expected number of cases based on the total incidence and the population in each scanning window, and then use the ratio of the actual number of cases inside and outside the window to the expected number of cases to calculate the relative risk (RR) and log-likelihood ratio (LLR). The window with the maximum LLR was defined as the most likely cluster, and other windows with smaller LLR, which were statistically significant, were defined as secondary clusters. The Markov Chain Monte Carlo (MCMC) method generates 999 simulation data sets, obtaining the probability P-value. The formula of LLR is:

$$
L L R=\ln \frac{L(z)}{L_{0}}=\ln \left(\frac{c}{n}\right)^{c}\left(\frac{C-c}{C-n}\right)^{c-c}
$$

$\mathrm{L}_{0}$ is the likelihood under the null hypothesis, which is always a constant for a certain data set, $L(z)$ is the likelihood of a certain area under the alternative hypothesis, $c$ is the actual number of cases in the window, and $\mathrm{n}$ is the window under the null hypothesis Expected number of cases, $\mathrm{C}$ is the total number of cases, C-c and C-n are the actual number of cases outside the window and the number of expected cases, respectively[35-37]. 
The space-time scan statistics analysis results are very sensitive to the parameter settings of the maximum spatial cluster size and the maximum length of the temporal scanning window[38]. Hjalmars suggested that $10 \%$ of the population at risk should be selected as the size of the maximum cluster[39]. A study recommended $15 \%$ of the number of people at risk as to the appropriate scale for the largest spatial scanning window[40]. In addition, the coverage of the largest cluster should not exceed $15 \%$ of the study area[41]. Across the country, HFMD had a peak activity in half a year. Southern China had two peak outbreaks of HFMD each year, and northern China had one peak outbreak each year[1]. Therefore, this study set $15 \%$ of the total population as the maximum cluster size, and the time frame of the scan analysis was set to one year to observe the cluster changes during the study.

SaTScan (version 10.0, Martin Kulldorff, Harvard Medical School, Boston, MA, USA and Information Management Services Inc, Calverton, MD, USA) was used to perform space-time scan statistics analysis of HFMD cases in 31 provinces of mainland China from 2009 to 2018. The analysis results were visualized on the map with ArcMap (version 10.2, ESRI Inc., Redlands, CA, USA).

\section{Results}

\section{Epidemiology Characteristics of HFMD Cases}

20,048,244 HFMD cases were reported from 2009 to 2018 in mainland China, with a median annual incidence of 143.22 per 100,000 (ranging from 87.01 to 205.06). A total of 3541 cases died with a case fatality rate of $0.18 \%$ o. Children 5 years and under were the main morbid population, accounting for $94.17 \%$ of all reported cases. The results of the reported cases grouped by age are shown in Table 1. In 2018 , the incidence of children who are 5 years and under was $92.76 \%$, the lowest level in 10 years.

Table 1. Distribution characteristics of HFMD cases by age group in Mainland China, 2009-2018.

\begin{tabular}{cccccccccccc}
\hline Age (year) & Total & 2009 & 2010 & 2011 & 2012 & 2013 & 2014 & 2015 & 2016 & 2017 & 2018 \\
\hline$<1$ & $9.59 \%$ & $7.26 \%$ & $8.34 \%$ & $8.85 \%$ & $9.38 \%$ & $13.04 \%$ & $7.74 \%$ & $11.29 \%$ & $8.64 \%$ & $12.03 \%$ & $9.40 \%$ \\
$1 \sim$ & $30.58 \%$ & $17.72 \%$ & $28.30 \%$ & $29.24 \%$ & $29.25 \%$ & $35.43 \%$ & $30.58 \%$ & $34.30 \%$ & $30.54 \%$ & $31.92 \%$ & $32.80 \%$ \\
$2 \sim$ & $22.52 \%$ & $29.08 \%$ & $24.64 \%$ & $24.55 \%$ & $23.73 \%$ & $21.45 \%$ & $23.76 \%$ & $21.65 \%$ & $21.30 \%$ & $19.67 \%$ & $18.87 \%$ \\
$3 \sim$ & $17.33 \%$ & $20.76 \%$ & $18.06 \%$ & $18.33 \%$ & $17.92 \%$ & $14.71 \%$ & $17.38 \%$ & $16.28 \%$ & $18.23 \%$ & $16.47 \%$ & $16.48 \%$ \\
$4 \sim$ & $9.50 \%$ & $12.15 \%$ & $9.77 \%$ & $9.39 \%$ & $9.58 \%$ & $7.23 \%$ & $9.77 \%$ & $7.72 \%$ & $10.57 \%$ & $9.33 \%$ & $10.03 \%$ \\
$5 \sim$ & $4.66 \%$ & $6.25 \%$ & $4.88 \%$ & $4.41 \%$ & $4.52 \%$ & $3.46 \%$ & $4.87 \%$ & $3.79 \%$ & $4.83 \%$ & $4.74 \%$ & $5.19 \%$ \\
$>5$ & $5.83 \%$ & $6.79 \%$ & $6.02 \%$ & $5.23 \%$ & $5.61 \%$ & $4.68 \%$ & $5.90 \%$ & $4.98 \%$ & $5.90 \%$ & $5.84 \%$ & $7.24 \%$ \\
\hline
\end{tabular}

Fig 1 shows the epidemic trend of the number of reported cases and annual incidence of HFMD in mainland China from 2009 to 2018. The number of reported cases and annual incidence of HFMD increased in even-numbered years (i.e.2010, 2012, 2014, 2016, 2018) and declined in odd-numbered years 
(i.e.2011, 2013, 2015, 2017) in mainland China, which demonstrated a phenomenon of increasing incidence in a two-year cycle from 2009 to 2014 and slightly decreasing incidence in a two-year cycle from 2015 to 2018. The incidence of HFMD peaked in 2014. Fig 2 shows the monthly distribution of the reported cases of HFMD in Mainland China from 2009 to 2018. Two peaks of infections were observed per year. The first peaks occurred from April to July, and the second occurred from September to November. The first peaks were higher than the second over the entire investigated period. In general, the result indicated that the incidence of HFMD was potentially seasonal and cyclical.

The spatial distribution of the incidence of HFMD in mainland China is shown in Fig 3, and dark color indicates high incidence. The incidence of HFMD varied greatly among provinces. Except for 2009, the incidence was higher in eastern and southern mainland China than in western and northeastern regions. The incidence of HFMD in the southern coastal provinces (Hainan, Guangxi, Guangdong) had been at the forefront of mainland China for many years. The inland province of Hunan had also been at a high incidence level for many years. Zhejiang had the highest incidence of HFMD in 2018. Adjacent to Zhejiang, Jiangsu, Shanghai, and Fujian also had high incidences. The western province of Chongqing showed the second-highest incidence this year. This result indicates the expansion of high-incidence areas.

\section{Spatial autocorrelation analysis}

The results of global spatial autocorrelation are shown in Table 2. The province-level of mainland China had a high global spatial autocorrelation from 2009 to 2018(global Moran's $I>0.395, P<0.001$ ). This result indicated that the spatial distribution of HFMD cases was non-random in mainland China, and there was a statistically significant positive correlation.

Table 2. Results of the global spatial autocorrelation analysis on HFMD incidence in Mainland China, 2009-2018. 


\begin{tabular}{llll} 
Year & Moran's I & Z-Value & $p$-Value \\
\hline 2009 & 0.587 & 4.972 & 0.001 \\
\hline 2010 & 0.395 & 3.588 & 0.001 \\
\hline 2011 & 0.419 & 4.778 & 0.001 \\
\hline 2012 & 0.500 & 4.868 & 0.001 \\
\hline 2013 & 0.485 & 4.857 & 0.001 \\
\hline 2014 & 0.476 & 4.581 & 0.001 \\
\hline 2015 & 0.432 & 4.899 & 0.001 \\
\hline 2016 & 0.503 & 4.694 & 0.001 \\
\hline 2017 & 0.547 & 5.386 & 0.001 \\
\hline 2018 & 0.534 & 4.664 & 0.001
\end{tabular}

According to the result of local spatial autocorrelation analysis combined with the LISA value, there were spatial correlation cluster maps for HFMD incidence in mainland China from 2009 to 2018 (Fig 4). In this study, positive spatial association (High-High cluster and Low-Low cluster) was found in mainland China every year from 2009 to 2018 . High-High clusters mainly appeared in a few provinces in southern China, including Guangdong, Guangxi, and Hunan. The results indicated that these provinces and their neighboring provinces have a high incidence of HFMD. The High-High cluster was found in new regions (Zhejiang and Fujian) in 2018, which meant that areas with a high incidence of HFMD have expanded. Low-Low cluster was only found in western and northern China, mainly Xinjiang, Xizang, Qinghai, and NeiMongol, which indicated that the incidence of HFMD is relatively low in western and northern China. Negative spatial associations (High-Low cluster and Low-High cluster) were scattered in mainland China and only found in a few inland provinces in a few years.

\section{Spatiotemporal Clusters Analysis}

Space-time scan analysis of HFMD data was conducted year by year in mainland China from 2009 to 2018. The results showed that the cases of HFMD were not randomly distributed in mainland China during the study period. Each year at least five significant spatiotemporal clusters were found, including the most likely cluster and secondary clusters. The red part on the map is the area of the most likely cluster (Fig 5). 
Table 3. The most likely spatiotemporal clusters of HFMD in mainland China, 2009-2018.

\begin{tabular}{|c|c|c|c|c|c|c|}
\hline Scas Teneframe & Chaster Tene & Cheser Provisce & Obsuerved Caves & Expected Canes & Relutive Resil & P-vilax \\
\hline 2009 & $\operatorname{Apc} .1 \sim \operatorname{Aug} 31$ & Tinajen, Beigng, Hebei, & 232685 & 7119993 & 384 & $\angle 001$ \\
\hline \multicolumn{7}{|c|}{ Shuisdoog } \\
\hline 2010 & $A p c .1 \sim \operatorname{dul} 11$ & Hainan, Grangri, Guagdoogs & 260017 & 71139.49 & 4.10 & $<001$ \\
\hline 2011 & May.1 Oce.31 & Hainin, Guangii, Grangdong & 363381 & 114465.30 & 3.75 & $<0.01$ \\
\hline 2012 & Ape.1 $\sim \operatorname{Seps} 30$ & Hainal, Guangi, Guangdoeg & 418634 & 129108.42 & 278 & -001 \\
\hline 2013 & May.1 $\sim$ Oct 31 & Haina, Guangri, Guagdoag & 492978 & 110465.11 & 5.74 & $<001$ \\
\hline 2014 & Ape 1 I Jul 31 & Hainas, Guingu, Guangdoeg & $\$ 39360$ & 11116760 & 5.78 & $<0.01$ \\
\hline 2015 & Mays - Oet 31 & Hainan, Geangri, Guangdoog & 521140 & 121473.15 & 3.44 & $<001$ \\
\hline 2016 & Aps. $1 \sim \operatorname{Jun} 30$ & Hainun, Guangii, Grangdong & 395400 & 79507.50 & 5.72 & $<0.01$ \\
\hline 2017 & May 1 -Oet 31 & Hainal, Guangi, Grangdoeg & 598793 & 124496.50 & 6.53 & 2001 \\
\hline 2018 & May.I $\sim \operatorname{Sep} .30$ & Sunghai, Jingera, Zxijung & 369979 & 120083.16 & 3.45 & $<001$ \\
\hline
\end{tabular}

Table 3 shows the cluster time, cluster province, relative risk, and other information of the most likely cluster from 2009 to 2018. In most years (2010-2017), the most likely cluster was in the southern coastal provinces, including Hainan, Guangxi, and Guangdong. The range of cluster time for these years was between April and October. The relative risk ranged from 3.75 to 6.53 , indicating that the incidence risk in the clusters was 3.75 to 6.53 times that of outside the gathering area in these years. In 2009, it was observed that the most likely cluster was in northern China, including Beijing, Tianjin, Hebei, and Shandong. The cluster time lasted from April to August. The relative risk was 3.84, indicating that the risk of incidence in the cluster was close to 4 times that outside the cluster. The most likely cluster was different from the past in 2018, which appeared in the southeast coastal areas, including Shanghai, Jiangsu, and Zhejiang. The cluster time was from May to September, and the relative risk was 3.45. Summarizing the results of space-time scan analysis from 2009 to 2018, we observed that the risk areas for HFMD were mainly southern coastal provinces (Hainan, Guangxi, Guangdong), followed by eastern coastal provinces (Zhejiang, Shanghai, Jiangsu, Fujian), which with a developed economy, high population density and strong population mobility. The high-risk time frame was at the end of spring, throughout the summer, and early autumn, when the climate is warm and humid. Although the central and southwest regions were not included in the most likely cluster, their relative risk was at the forefront for many years, and these provinces need to be paid attention to.

\section{Discussion}

Since HFMD was listed as a notifiable infection in China, it has caused an enormous disease burden. It has a severe impact on the health of children under five years. The launch of the vaccine in 2016 might change the epidemiological character of HFMD in mainland China. Using GIS technology to analyze the spatiotemporal distribution patterns of HFMD in mainland China will help further to explore the high-risk areas and times of the disease and provide a basis for developing more effective HFMD prevention and control policies.

This study found that the incidence of HFMD in mainland China showed an overall downward trend after 2014, which may be related to HFMD vaccines launched against EV-A71 enterovirus in mainland China in 
2016[26, 42]. However, the decline in the incidence of HFMD was not large. The incidence of HFMD in Mainland China was 169.41 per 100,000 in 2018, which was only lower than the incidence in 2014 and 2016, higher than Thailand's incidence rate of 102.51/100,000[43] and Malaysia's incidence rate of $138.6 / 100,000$ [44]. So, the incidence of HFMD was still at a high level in mainland China in 2018. There may be three reasons for this. On the one hand, the monovalent inactivated EV-A71 vaccines cannot provide protection against non-EV-A71 virus infections of HFMD[45]. On the other hand, due to the stable birth rate and no cross-protection of alternate serotypes, the previous HFMD epidemics did not reduce the susceptible persons in the population probably[46]. In addition, the vaccines of HFMD have not been included in the National Immunization Program in China, which means that parents need to vaccinate their children at their own expense and voluntarily. Hence, the vaccination coverage rate was not high[47]. A study found that the annual EV-A71 vaccination coverage rate of children under 5 years old ranged from 5.53\% to 15.01\% in Yunnan from 2016 to 2019[48]. In Guangdong, the coverage rate of EV-A71 vaccination among the 6-month to 5 -year children was $2.67 \%$ in 2016 and $10.07 \%$ in 2017[49]. A study in Chengdu showed that the EV-A71 vaccination coverage rate should reach $94.0 \%$ to end the EV-A71related HFMD transmission[50]. Therefore, efforts should be made to develop multivalent vaccines against major enteroviruses and increase the vaccination rate in the future.

The study results suggested the cyclical and seasonal pattern of the HFMD epidemics. The HFMD epidemic took two years as a cycle. This cyclical pattern was observed in many countries, and there was evidence that this cyclicality is related to the time required to accumulate enough susceptible children in the population[7, 51, 52]. In this study, the seasonal pattern of HFMD was reflected in the peak incidence of twice a year, the first peak occurred in April to July, and the second peak occurred from September to November in mainland China. Seasonal patterns of HFMD have also been observed in other countries. HFMD usually breaks out from June to August in Thailand[53], cases of HFMD increased during the rainy season in Vietnam[54]. However, the seasonal patterns observed in temperate, tropical, and subtropical Asia are different[55]. In China, the seasonal characteristics of the south and the north were also inconsistent[56]. In mainland China, the first peak of each year may be related to climate factors such as temperature, humidity, and atmospheric pressure. Appropriate climatic conditions are conducive to the reproduction and survival of pathogens. Warm temperature promotes children's activities and mutual contact, thereby promoting the transmission of pathogens. Although the temperature and humidity are still high in August, the school has summer vacation, which reduces the gathering and contact of students, so the incidence is reduced. September coincides with the end of summer and the beginning of autumn, so the temperature and humidity are still high. The second peak may be due to the opening of kindergarten in September to increase the contact between children, thereby increasing the possibility of cluster cases[24, 44, 56-59].

We also found that children under 5 years are the main morbid population, accounting for $94.17 \%$ of all reported cases. The result was consistent with other research's findings in other countries and China[1, 7 , 43 , 47]. The low incidence of children over 5 years old maybe because they have had HFMD before, thereby protecting them and their peers from $\operatorname{HFMD}[60,61]$. In 2018, the incidence of children aged 5 and under was the lowest level in 10 years, which may be related to the increase in the cumulative coverage of 
vaccines[26]. Therefore, we call for attention to children in this age range, vaccinate them as soon as possible, and introduce the HFMD vaccine into the routine immunization program.

The global Moran's I for 2009-2018 ranged from 0.395 to 0.587 . The global spatial autocorrelation analysis results showed that the spatial distribution of HFMD cases was non-random in mainland China and tended to cluster at the provincial level, which was the same as the analysis result for 2008-2011 by Xiao[62]. Combined with the incidence distribution map (Fig 3), we found that the incidence of HFMD was quite different between the southern and northern of China. Overall, the incidence of south China was much higher than that in north China. This phenomenon has also been found in other studies[1,23, 24, 63]. Meteorological factors are a fundamental reason for the difference in incidence between south and north China. South China is mostly in high temperature, high humidity, and rainy weather conditions, which to a certain extent promotes the reproduction, survival, and spread of the pathogens of HFMD[23, 64]. Each year, there was only one peak incidence in late spring and early summer (May-July) in the northern region. At the same time, there were two peak incidences in April-June and September-November in the southern region[1, 25, 56].

The most likely cluster appeared in southern coastal provinces (Guangxi, Guangdong, Hainan) from 2010 to 2017. The results of local spatial autocorrelation analysis and spatiotemporal clusters analysis indicated that the southern coastal provinces (Guangxi, Guangdong, Hainan) were the areas with a high incidence of HFMD in China. Their incidence had been at the highest level in mainland China for many years, the same as previous research results[23,65,66]. The climate of these provinces is characterized by short and mild winter, while summer is long, hot, and very humid. In addition to climatic factors, the high incidence in southern coastal provinces may also be due to the high population density, strong population mobility, developed economy, and more frequent communication and close contact between individuals, which led to a higher risk of spreading $\operatorname{HFMD}[44,58,65-67]$. The cluster time ranged between April and October, which was consistent with the peak time of HFMD in mainland China[1]. In 2017, the relative risk of the most likely cluster (Hainan, Guangxi, Guangdong) was 6.53, which was higher than before. Because the incidence of HFMD had decreased in other provinces, while the incidence of Hainan, Guangxi, and Guangdong were still at a high level, the low coverage rate of the vaccine may be one of the reasons. In 2017, the cumulative coverage rate of EV-A71 vaccine among 6-month to 5-year children was $9.26 \%$ in Guangxi[26] and $10.07 \%$ in Guangdong[49], while it was $18.94 \%$ in Yunnan[48] and $15 \%$ in Beijing[68]. In addition, some studies have found that non-EV-A71 enteroviruses were the dominant serotype in Guangxi in 2017, while the monovalent EV-A71 vaccine cannot generate cross-immunity against other enterovirus infections[26, 58,69]. In 2018, the southern coastal provinces (Guangxi, Guangdong, Hainan) were no longer the most likely cluster. The decline in the incidence of HFMD was large in these three provinces, and the incidence had reduced to the lowest level during the study period, which was 258.10 per 100,000 in Hainan and 354.28 per 100,000 in Guangxi, 265.23 per 100,000 in Guangdong. This result may be related to the increase in vaccine coverage. Since Guangxi implemented comprehensive prevention and control measures focusing on promoting the EV-A71 vaccine among susceptible populations, the vaccine coverage rate of susceptible children had approached $30 \%$ in Guangxi in 2018, and the EV-A71-related incidence had declined[26, 69]. The same finding was also 
found in the study of Guangdong[58, 70]. For this reason, it is necessary to expand the HFMD vaccination program.

According to the results of spatiotemporal clusters analysis, we found that three southeast coastal provinces (Shanghai, Jiangsu, Zhejiang) were included in secondary cluster 1 from 2010 to 2016. The relative risk of this cluster was only second to the most likely cluster. This result indicated that these provinces were areas with a high incidence of HFMD, and they had a higher incidence than the northern and inland provinces. These provinces have a subtropical climate, so they are tended to be hotter and more humid. Furthermore, they have a higher population density and a larger migratory population. These factors are the main reason for the high incidence of HFMD[71]. In 2018, these provinces constituted the new most likely cluster, and the cluster time ranged between May and September. We also discovered that the new High-High cluster area appeared in Jiangsu in 2018 through local spatial autocorrelation analysis. Both results suggested that the incidence of HFMD in Jiangsu and its neighboring Shanghai and Zhejiang was higher than that of other provinces in 2018, which was found in other studies[72, 73]. However, the incidence of the previous most likely cluster (Hainan, Guangxi, Guangdong) had decreased, so the southeast coastal provinces (Shanghai, Jiangsu, Zhejiang) became the new most likely cluster. In 2018 , the incidence in Zhejiang and Jiangsu reached the highest value after the vaccine was launched, which was 436.02 per 100,000 in Zhejiang and 237.50 per 100,000 in Jiangsu, and Zhejiang had the highest incidence in the country. EV-A71 vaccine coverage rate had increased year by year to $24.05 \%$ in Zhejiang, higher than $19.4 \%$ in Guangdong[74]. The proportion of EV-A71 positive cases declined from 22.6\% in 2017 to 3.3\% in 2018 in Zhejiang[75]. Another study found that CV-A16 and CV-A6 became the primary pathogens of HFMD in Jiangsu, and the cases caused by these two pathogens accounted for more than $96 \%$ of all cases[76]. Therefore, the peak incidence of HFMD in 2018 may be related to other serotypes becoming dominant. Even large-scale vaccination of the EV-A71 vaccine would not greatly reduce the number of cases[26, 76]. This result suggested that the dominant pathogens in different regions and at different times are various. Therefore, it is necessary to monitor the enterovirus genotypes of HFMD cases in various places and promote HFMD multivalent vaccines.

Compared with studies in various provinces[65, 66, 77, 78], we found that clusters were not only distributed in one province but also cross-linked to multiple provinces, which showed the importance of cooperation between provinces to prevent and control the spread of HFMD[25]. This strong spatial and temporal correlation of HFMD was also found in other studies, which may be related to meteorological factors. Because meteorological factors promote the prevalence of HFMD, the meteorological conditions in adjacent areas and adjacent time points are similar[71].

The southern inland province Hunan was included in the High-High cluster areas in 2014 and 2016-2018. Although Hunan had not appeared in the most likely cluster, the results of spatiotemporal clusters analysis showed that Hunan was one of the high-risk areas for HFMD. It was observed to have a high incidence, which was also found in other studies[79]. Because Hunan has a subtropical monsoon climate with abundant heat, concentrated rainfall, and high humidity, in addition to high population density and high levels of migration, both suitable natural and social environments have promoted the development 
and spread of enteroviruses in Hunan[78, 80]. This result suggested that the HFMD epidemic in Hunan was worthy of attention.

For the prevention and control strategy of HFMD, we make the following suggestions: First, the prevention and control of HFMD should focus on southeastern and southern coastal provinces in mainland China, especially from April to September each year. Attention should also be paid to the southern inland provinces. Moreover, regarding the strong spatial and temporal association of HFMD in neighboring provinces, the provinces should strengthen cooperation and joint control to avoid a widespread HFMD across provinces. In addition, it is necessary to advance the EV-A71 vaccination plan and expand the vaccine coverage, especially in coastal areas of China. As the dominant pathogens have changed, future research should analyze the spatial-temporal distribution characteristics of different pathogens of HFMD and develop multivalent HFMD vaccines as soon as possible.

The main advantage of our research is using Chinese CDC's disease surveillance data, so the authenticity and completeness of the data are guaranteed. In addition, our study used spatiotemporal clusters analysis that combines time and space, revealing the temporal and spatial distribution patterns of HFMD at the province level in mainland China. We set $15 \%$ of the risk population as the maximum cluster size in the analysis, avoiding the most likely cluster containing low-risk regions and thus larger than the actual cluster. In this study, the data of HFMD cases were scanned year by year, and the trend of the most likely cluster could be found. As far as we know, it is the first study to analyze the spatiotemporal clusters of HFMD in the whole of mainland China after the HFMD vaccines were launched in China.

The limitations of this study are worth mentioning. First, although Chinese CDC disease surveillance data could ensure the authenticity and completeness of the data to a certain extent, it may not avoid the differences in the quality of case reports in the surveillance systems of different regions and the underreporting of cases caused by mild cases that do not go to the hospital for treatment. In addition, the outbreak of COVID-19 in 2019 may change the spatial and temporal distribution pattern of HFMD in China. However, we have not obtained HFMD data after 2019. The incidence data of HFMD was at the provincial level. More accurate results may be attained if using smaller spatial scale (such as district, county) information. On the other hand, the spatial scanning window used to detect the cluster was circular, while the geographic shape was irregular, which may not represent the actual shapes of the clusters.

\section{Conclusions}

In summary, changes in the spatiotemporal cluster of HFMD after the launch of EV-A71 vaccines were observed at the province level in mainland China from 2016 to 2018. The most likely cluster appeared in the southern coastal provinces (Guangxi, Guangdong, Hainan) from 2010 to 2017 and in the southeastern coastal provinces (Shanghai, Jiangsu, Zhejiang) in 2018. The range of cluster time was between April and October. The spatiotemporal cluster of HFMD may be related to climate factors, population density, vaccine coverage, enterovirus genotypes. Our research results provide information for 
further determining the high-risk areas of HFMD after the vaccines were launched and the development of more effective HFMD prevention and control policies.

\section{Abbreviations}

HFMD: Hand, foot, and mouth disease

EV-A71: Enterovirus A71

CV-A16: Coxsackievirus A16

Chinese CDC: Chinese Center for Disease Control and Prevention

NIDRIS: Notifiable Infectious Disease Reporting System

NBSC: National Bureau of Statistics of China

NGCC: National Geomatics Center of China

LISA: Local indicator of spatial association

RR: relative risk

MCMC: Markov Chain Monte Carlo

\section{Declarations}

Ethics approval and consent to participate

Not applicable.

\section{Consent for publication:}

Not applicable.

\section{Availability of data and materials}

The data used in this study are owned by public health science data center given by the Chinese Center for Diseases Control and Prevention (Chinese CDC). The data are publicly available and are available to researchers by visiting the official website of the public health science data center given by the Chinese CDC(https://www.phsciencedata.cn/Share/ky_sjml.jsp?id=b9c93769-3e0f-413a-93c1-027d2009d8bc) [29].

\section{Competing interests}

The authors declare they have no competing interests. 


\section{Funding}

This study was funded by the projects of the Basic Data Investigation Project on Incidence and Risk Factors of Intracerebral Hemorrhage in Central China (grant number 2018FY100900) and Drawing of Feature Map and Spatial Heterogeneity Analysis of Intracerebral Hemorrhage in Central China (grant number 2018FY100903). The funder had no role in the study design, data collection and analysis, interpretation of data, and writing the manuscript.

\section{Authors' contributions}

Y.W. and T.W. performed the analysis and drafted the manuscript. S.D. undertook the download and management of the data. M.Z. and S.W. critically revised the manuscript. J.S. led the work and reviewed the manuscript. All authors read and approved the final manuscript.

\section{Acknowledgments}

We would like to sincerely thank the Chinese Center for Disease Control and Prevention, National Bureau of Statistics of China, and National Geomatics Center of China for their support with date access.

\section{References}

1. Xing WJ, Liao QH, Viboud C, Zhang J, Sun JL, Wu JT, Chang ZR, Liu FF, Fang VJ, Zheng YD et al: Hand, foot, and mouth disease in China, 2008-12: an epidemiological study. Lancet Infectious Diseases 2014, 14(4):308-318.

2. Li XW, Ni X, Qian SY, Wang Q, Jiang RM, Xu WB, Zhang YC, Yu GJ, Chen Q, Shang YX et al: Chinese guidelines for the diagnosis and treatment of hand, foot and mouth disease (2018 edition). World Journal of Pediatrics 2018, 14(5):437-447.

3. Schoffel N, Klingelhofer D, Braun M, Bruggmann D, Groneberg DA: Hand, foot and mouth disease. A review of the literature. Zentralblatt Fur Arbeitsmedizin Arbeitsschutz Und Ergonomie 2018, 68(2):91-93.

4. Ooi MH, Wong SC, Lewthwaite P, Cardosa MJ, Solomon T: Clinical features, diagnosis, and management of enterovirus 71. Lancet Neurology 2010, 9(11):1097-1105.

5. Yi LN, Lu J, Kung HF, He ML: The virology and developments toward control of human enterovirus 71. Critical Reviews in Microbiology 2011, 37(4):313-327.

6. Solomon T, Lewthwaite P, Perera D, Cardoso MJ, McMinn P, Ooi MH: Virology, epidemiology, pathogenesis, and control of enterovirus 71. Lancet Infectious Diseases 2010, 10(11):778-790.

7. NikNadia NMN, Sam IC, Rampal S, WanNorAmalina WMZ, NurAtifah G, Verasahib K, Ong CC, MohdAdib M, Chan YF: Cyclical Patterns of Hand, Foot and Mouth Disease Caused by Enterovirus A71 in 
Malaysia. Plos Neglected Tropical Diseases 2016, 10(3).

8. Lim CTK, Jiang L, Ma S, James L, Ang LW: Basic reproduction number of coxsackievirus type A6 and $\mathrm{A} 16$ and enterovirus 71: estimates from outbreaks of hand, foot and mouth disease in Singapore, a tropical city-state. Epidemiology and Infection 2016, 144(5):1028-1034.

9. Noisumdaeng P, Korkusol A, Prasertsopon J, Sangsiriwut K, Chokephaibulkit K, Mungaomklang A, Thitithanyanont A, Buathong R, Guntapong R, Puthavathana P: Longitudinal study on enterovirus A71 and coxsackievirus A16 genotype/subgenotype replacements in hand, foot and mouth disease patients in Thailand, 2000-2017. International Journal of Infectious Diseases 2019, 80:84-91.

10. Nhan LNT, Khanh TH, Hong NTT, Van HMT, Nhu LNT, Ny NTH, Nguyet LA, Thanh TT, Anh NT, Hang VTT et al: Clinical, etiological and epidemiological investigations of hand, foot and mouth disease in southern Vietnam during 2015-2018. Plos Neglected Tropical Diseases 2020, 14(8).

11. Gonzalez G, Carr MJ, Kobayashi M, Hanaoka N, Fujimoto T: Enterovirus-Associated Hand-Foot and Mouth Disease and Neurological Complications in Japan and the Rest of the World. International Journal of Molecular Sciences 2019, 20(20).

12. Fu XM, Wan ZZ, Li YP, Hu YH, Jin X, Zhang CY: National Epidemiology and Evolutionary History of Four Hand, Foot and Mouth Disease-Related Enteroviruses in China from 2008 to 2016. Virologica Sinica 2020, 35(1):21-33.

13. Shi C, Liu J, Shi P, Ji H, Shen Y, Qian YH: Epidemiological characteristics and influential factors of hand, foot, and mouth disease reinfection in Wuxi, China, 2008-2016. Bmc Infectious Diseases 2018, 18.

14. Samphutthanon R, Tripathi NK, Ninsawat S, Duboz R: Spatio-Temporal Distribution and Hotspots of Hand, Foot and Mouth Disease (HFMD) in Northern Thailand. International Journal of Environmental Research and Public Health 2014, 11(1):312-336.

15. Wong SSY, Yip CCY, Lau SKP, Yuen KY: Human enterovirus 71 and hand, foot and mouth disease. Epidemiology and Infection 2010, 138(8):1071-1089.

16. Podin Y, Gias ELM, Ong F, Leong YW, Yee SF, Yusof MA, Perera D, Teo B, Wee TY, Yao SC et al: Sentinel surveillance for human enterovirus 71 in Sarawak, Malaysia: lessons from the first 7 years. $B m c$ Public Health 2006, 6.

17. Nguyen HX, Chu C, Nguyen HLT, Nguyen HT, Do CM, Rutherford S, Phung D: Temporal and spatial analysis of hand, foot, and mouth disease in relation to climate factors: A study in the Mekong Delta region, Vietnam. Science of the Total Environment 2017, 581:766-772.

18. Sham NM, Krishnarajah I, Ibrahim NA, Lye MS: Temporal and spatial mapping of hand, foot and mouth disease in Sarawak, Malaysia. Geospatial Health 2014, 8(2):503-507. 
19. Lee CCD, Tang JH, Hwang JS, Shigematsu M, Chan TC: Effect of Meteorological and Geographical Factors on the Epidemics of Hand, Foot, and Mouth Disease in Island-Type Territory, East Asia. Biomed Research International 2015, 2015.

20. Zhang XX, Xu CD, Xiao GX: Spatial heterogeneity of the association between temperature and hand, foot, and mouth disease risk in metropolitan and other areas. Science of the Total Environment 2020, 713.

21. Wang JF, Guo YS, Christakos G, Yang WZ, Liao YL, Li ZJ, Li XZ, Lai SJ, Chen HY: Hand, foot and mouth disease: spatiotemporal transmission and climate. International Journal of Health Geographics 2011, 10.

22. Wang JJ, Cao ZD, Zeng DD, Wang QY, Wang XL, Qian HK: Epidemiological Analysis, Detection, and Comparison of Space-Time Patterns of Beijing Hand-Foot-Mouth Disease (2008-2012). Plos One 2014, 9(3).

23. Chen B, Yang Y, Xu XF, Zhao HX, Li Y, Yin S, Chen YQ: Epidemiological characteristics of hand, foot, and mouth disease in China A meta-analysis. Medicine 2021, 100(20).

24. Huang RF, Wei JT, Li ZW, Gao ZG, Mahe M, Cao WC: Spatial-temporal mapping and risk factors for hand foot and mouth disease in northwestern inland China. Plos Neglected Tropical Diseases 2021, 15(3).

25. Wang C, Li X, Zhang YJ, Xu Q, Huang FF, Cao K, Tao LX, Guo J, Gao Q, Wang W et al: Spatiotemporal Cluster Patterns of Hand, Foot, and Mouth Disease at the County Level in Mainland China, 2008-2012. Plos One 2016, 11(1).

26. Wang J, Jiang LN, Zhang C, He WT, Tan Y, Ning CAY: The changes in the epidemiology of hand, foot, and mouth disease after the introduction of the EV-A71 vaccine. Vaccine 2021, 39(25):3319-3323.

27. Liu LL, Wang L, Qi C, Zhu YC, Li CY, Jia Y, She KL, Liu TX, Zhang Y, Cui F et al: Epidemiological characteristics and spatiotemporal analysis of hand-foot-mouth diseases from 2010 to 2019 in Zibo city, Shandong, China. Bmc Public Health 2021, 21(1).

28. Ding L, Zhang N, Zhu B, Liu JL, Wang X, Liu F, Mao Y: Spatiotemporal characteristics and meteorological determinants of hand, foot and mouth disease in Shaanxi Province, China: a county-level analysis. Bmc Public Health 2021, 21(1).

29. Public Health Science Data Center.[https://www.phsciencedata.cn/Share/ky_sjml.jsp? id=b9c93769-3e0f-413a-93c1-027d2009d8bc] Accessed 20 November 2021

30. National Bureau of Statistics of the People's Republic of China.[https://data.stats.gov.cn] Accessed 20 November 2021 
31. National Geomatics Center of China.[http://www.ngcc.cn/ngcc/html/1/index.html] Accessed 20 November 2021

32. Pfeiffer DU, Robinson TP, Stevenson M, Stevens KB, Rogers DJ, Clements ACA: Spatial Analysis in Epidemiology; 2008.

33. Zhu B, Fu Y, Liu JL, Mao Y: Notifiable Sexually Transmitted Infections in China: Epidemiologic Trends and Spatial Changing Patterns. Sustainability 2017, 9(10).

34. Anselin L: LOCAL INDICATORS OF SPATIAL ASSOCIATION - LISA. Geographical Analysis 1995, 27(2):93-115.

35. Jones SG, Kulldorff M: Influence of Spatial Resolution on Space-Time Disease Cluster Detection. Plos One 2012, 7(10).

36. Kulldorff M, Feuer EJ, Miller BA, Freedman LS: Breast cancer clusters in the northeast United States: A geographic analysis. Am J Epidemiol 1997, 146(2):161-170.

37. Yang SQ, Fang ZG, Lv CX, An SY, Guan P, Huang DS, Wu W: Spatiotemporal cluster analysis of COVID-19 and its relationship with environmental factors at the city level in mainland China.

Environmental Science and Pollution Research 2021.

38. Ma Y, Yin F, Zhang T, Zhou XA, Li XS: Selection of the Maximum Spatial Cluster Size of the Spatial Scan Statistic by Using the Maximum Clustering Set-Proportion Statistic. Plos One 2016, 11(1).

39. Hjalmars U, Kulldorff M, Gustafsson G, Nagarwalla N: Childhood leukaemia in Sweden: using GIS and a spatial scan statistic for cluster detection. Statistics in medicine 1996, 15(7-9):707-715.

40. Tango T, Takahashi K: A flexibly shaped spatial scan statistic for detecting clusters. International journal of health geographics 2005, 4:11.

41. Rao HX, Shi XY, Zhang X: Using the Kulldorff's scan statistical analysis to detect spatio-temporal clusters of tuberculosis in Qinghai Province, China, 2009-2016. Bmc Infectious Diseases 2017, 17.

42. Liu ZX, Tian J, Wang Y, Li YX, Liu-Helmersson J, Mishra S, Wagner AL, Lu YH, Wang WB: The burden of hand, foot, and mouth disease among children under different vaccination scenarios in China: a dynamic modelling study. Bmc Infectious Diseases 2021, 21(1).

43. Thammasonthijarern N, Kosoltanapiwat N, Nuprasert W, Sittikul P, Sriburin P, Pan-ngum W, Maneekan P, Hataiyusuk S, Hattasingh W, Thaipadungpanit J et al: Molecular Epidemiological Study of Hand, Foot, and Mouth Disease in a Kindergarten-Based Setting in Bangkok, Thailand. Pathogens 2021, 10(5). 
44. Fong SY, Mori D, Rundi C, Yap JF, Jikal M, Abd Latip A, Johnny V, Ahmed K: A five-year retrospective study on the epidemiology of hand, foot and mouth disease in Sabah, Malaysia. Scientific Reports 2021, 11(1).

45. Fang CY, Liu CC: Novel strategies for the development of hand, foot, and mouth disease vaccines and antiviral therapies. Expert Opinion on Drug Discovery.

46. Yang BY, Liu FF, Liao QH, Wu P, Chang ZR, Huang J, Long L, Luo L, Li Y, Leung GM et al: Epidemiology of hand, foot and mouth disease in China, 2008 to 2015 prior to the introduction of EV-A71 vaccine. Eurosurveillance 2017, 22(50):38-47.

47. Zhang Z, Liu Y, Liu FF, Ren MR, Nie TR, Cui JZ, Chang ZR, Li ZJ: Basic Reproduction Number of Enterovirus 71 and Coxsackievirus A16 and A6: Evidence From Outbreaks of Hand, Foot, and Mouth Disease in China Between 2011 and 2018. Clinical Infectious Diseases 2021, 73(9):E2552-E2559.

48. Jiang L, Jiang HC, Tian X, Xia XS, Huang T: Epidemiological characteristics of hand, foot, and mouth disease in Yunnan Province, China, 2008-2019. Bmc Infectious Diseases 2021, 21(1).

49. Fen Y, LIANG W-j, SUN L-m: Innoculation of enterorirus $\mathbf{7 1}$ vaccine and incidence of hand-footmouth disease in Guangdong province. China Public Health 2020, 36(3):351-354.

50. Han Y, Chen Z, Zheng K, Li X, Kong J, Duan X, Xiao X, Guo B, Luan R, Long L: Epidemiology of Hand, Foot, and Mouth Disease Before and After the Introduction of Enterovirus 71 Vaccines in Chengdu, China, 2009-2018. The Pediatric Infectious Disease Journal 2020, 39(10):969-978.

51. Xu LL, Shi Y, Rainey JJ, Zhang ZJ, Zhang HY, Zhao JH, Li YH, Rao HX, Li YM, Liao QH et al: Epidemiological features and spatial clusters of hand, foot, and mouth disease in Qinghai Province, China, 2009-2015. Bmc Infectious Diseases 2018, 18.

52. Sabanathan S, Tan LV, Thwaites L, Wills B, Qui PT, van Doorn HR: Enterovirus 71 related severe hand, foot and mouth disease outbreaks in South-East Asia: current situation and ongoing challenges. Journal of Epidemiology and Community Health 2014, 68(6):500-502.

53. Verma S, Razzaque MA, Sangtongdee U, Arpnikanondt C, Tassaneetrithep B, Arthan D, Paratthakonkun C, Soonthornworasiri N: Hand, Foot, and Mouth Disease in Thailand: A Comprehensive Modelling of Epidemic Dynamics. Computational and Mathematical Methods in Medicine 2021, 2021.

54. Pham HV, Phan UTN, Pham ANQ: Meteorological factors associated with hand, foot and mouth disease in a Central Highlands province in Viet Nam: an ecological study. Western Pacific Surveillance and Response 2019, 10(4).

55. Koh WM, Bogich T, Siegel K, Jin J, Chong EY, Tan CY, Chen MIC, Horby P, Cook AR: The Epidemiology of Hand, Foot and Mouth Disease in Asia A Systematic Review and Analysis. Pediatric Infectious Disease Journal 2016, 35(10):E285-E300. 
56. Yu CC, Xu CJ, Li YH, Yao SQ, Bai YC, Li JZ, Wang L, Wu WD, Wang YB: Time Series Analysis and Forecasting of the Hand-Foot-Mouth Disease Morbidity in China Using An Advanced Exponential Smoothing State Space TBATS Model. Infection and Drug Resistance 2021, 14:2809-2821.

57. Hong SZ, Liu FF, Bauer C, Chen Y, Tu W, Zhang J, Hu J, Zhang WY, Hu Y, Lynn HS et al: Intra-area factors dominate the spatio-temporal transmission heterogeneity of hand, foot, and mouth disease in China: A modelling study. Science of the Total Environment 2021, 775.

58. Du ZC, Huang Y, Lawrence WR, Xu JX, Yang ZC, Lu JY, Zhang ZB, Hao YT: Leading Enterovirus Genotypes Causing Hand, Foot, and Mouth Disease in Guangzhou, China: Relationship with Climate and Vaccination against EV71. International Journal of Environmental Research and Public Health 2021, 18(1).

59. Zhao JJ, Hu XY: The complex transmission seasonality of hand, foot, and mouth disease and its driving factors. Bmc Infectious Diseases 2019, 19.

60. Chew SP, Chong SL, Barbier S, Matthew A, Lee JH, Chan YH: Risk factors for severe hand foot mouth disease in Singapore: a case control study. Bmc Infectious Diseases 2015, 15.

61. Shah VA, Chong CY, Chan KP, Ng W, Ling AE: Clinical characteristics of an outbreak of hand, foot and mouth disease in Singapore. Annals Academy of Medicine Singapore 2003, 32(3):381-387.

62. Xiao G-x, Hu Y-h, Ma J-q, Hao Y-t, Wang X-f, Zhang Y-j, Yu S-c: Spatial clustering and changing trend of hand-foot-mouth disease during 2008-2011 in China. Zhonghua liu xing bing xue za zhi = Zhonghua liuxingbingxue zazhi 2012, 33(8):808-812.

63. Zhao JJ, Li XM: Determinants of the Transmission Variation of Hand, Foot and Mouth Disease in China. Plos One 2016, 11(10).

64. Pan Q, Liu FF, Zhang JY, Zhao X, Hu YF, Fan CN, Yang F, Chang ZR, Xiao X: Regional-level risk factors for severe hand-foot-and-mouth disease: an ecological study from mainland China.

Environmental Health and Preventive Medicine 2021, 26(1).

65. Deng T, Huang Y, Yu SC, Gu J, Huang CR, Xiao GX, Hao YT: Spatial-Temporal Clusters and Risk Factors of Hand, Foot, and Mouth Disease at the District Level in Guangdong Province, China. Plos One 2013, 8(2).

66. Xie YH, Chongsuvivatwong V, Tang ZZ, McNeil EB, Tan Y: Spatio-Temporal Clustering of Hand, Foot, and Mouth Disease at the County Level in Guangxi, China. Plos One 2014, 9(2).

67. Huang JX, Wang JF, Li ZJ, Wang Y, Lai SJ, Yang WZ: Visualized Exploratory Spatiotemporal Analysis of Hand-Foot-Mouth Disease in Southern China. Plos One 2015, 10(11). 
68. Wang XL, An ZJ, Huo D, Jia L, Li J, Yang Y, Liang ZC, Wang QY, Wang HQ: Enterovirus A71 vaccine effectiveness in preventing enterovirus A71 infection among medically-attended hand, foot, and mouth disease cases, Beijing, China. Human Vaccines \& Immunotherapeutics 2019, 15(5):1183-1190.

69. Ning K, Yu J, Jing W, Min C, Chao Z, Min-mei C: Epidemiologic characteristics and pathogenic surveillance results of hand, foot and mouth disease in Guangxi, 2010-2018. China Tropical Medicine 2021, 21(10):927-931+938.

70. Du ZC, Huang Y, Bloom MS, Zhang ZB, Yang ZC, Lu JY, Xu JX, Hao YT: Assessing the vaccine effectiveness for hand, foot, and mouth disease in Guangzhou, China: a time-series analysis. Human Vaccines \& Immunotherapeutics 2021, 17(1):217-223.

71. Lau SYF, Chen EF, Mohammad KN, Cai J, Wang MHT, Zee BCY, Zhao S, Chong KC, Wang XX: Ambient temperature and relative humidity as possible drivers of the hand, foot, and mouth disease epidemics in Zhejiang Province, China. Atmospheric Environment 2021, 244.

72. Nie TR, Cui JZ, Ren MR, Liu FF, Sun JL, Zhang J, Chang ZR: Application of moving epidemic method in establishing epidemic intensity threshold of hand, foot, and mouth disease in southern China. Zhonghua liu xing bing xue za zhi = Zhonghua liuxingbingxue zazhi 2020, 41(7):1047-1053.

73. Ji H, Fan H, Lu PX, Zhang XF, Ai J, Shi C, Huo X, Bao CJ, Shan J, Jin Y: Surveillance for severe hand, foot, and mouth disease from 2009 to 2015 in Jiangsu province: epidemiology, etiology, and disease burden. Bmc Infectious Diseases 2019, 19.

74. Ye LX, Chen JP, Fang T, Ma R, Wang JM, Pan XQ, Dong HJ, Xu GZ: Vaccination coverage estimates and utilization patterns of inactivated enterovirus 71 vaccine post vaccine introduction in Ningbo, China. Bmc Public Health 2021, 21(1).

75. Qin C, Yu-lu F, Hui W, Li-ping W, Zi-ping M, Chang-zheng D: Pathogenic Characteristics of Hand Foot and Mouth Disease in Zhejiang Province from 2017 to 2019. CHINESE PRIMARY HEALTH CARE 2021, 35(11):51-55.

76. Liu S-y, Huo Y-q, Gu M-r, Gao F, Mao Q-y, Jiang W, Bian L-I: Molecular epidemic characteristics of pathogen of hand, foot and mouth disease in Guangdong and Jiangsu Provinces, China from 2018 to 2019. Chinese Journal of Biologicals 2021, 34(2):186-191.

77. Qi L, Tang WG, Zhao H, Ling H, Su K, Zhao H, Li Q, Shen T: Epidemiological Characteristics and Spatial-Temporal Distribution of Hand, Foot, and Mouth Disease in Chongqing, China, 2009-2016. International Journal of Environmental Research and Public Health 2018, 15(2).

78. Wu XR, Hu SX, Kwaku AB, Li Q, Luo KW, Zhou Y, Tan HZ: Spatio-temporal clustering analysis and its determinants of hand, foot and mouth disease in Hunan, China, 2009-2015. Bmc Infectious

Diseases 2017, 17. 
79. Wei J-H, Wu R, Xing D-X, Shu J, Hu S-X, Qin J-B: Epidemiological characteristics and spatial epidemiology of hand-foot-mouth disease in Hunan Province, China, from 2008 to 2019. Zhongguo dang dai er ke za zhi = Chinese journal of contemporary pediatrics 2021, 23(11):1141-1148.

80. Liao YL, Xu B, Liu XC, Wang JF, Hu SX, Huang W, Luo KW, Gao LD: Using a Bayesian belief network model for early warning of death and severe risk of HFMD in Hunan province, China. Stochastic Environmental Research and Risk Assessment 2018, 32(6):1531-1544.

\section{Figures}

\section{Figure 1}

Annual incidence and number of cases of HFMD in mainland China,2009-2018.

Figure 2

Monthly distribution of HFMD cases in mainland China, 2009-2018.

\section{Figure 3}

Spatial distribution of HFMD incidence in mainland China, 2009-2018.

\section{Figure 4}

Spatial correlation cluster maps of HFMD incidence in mainland China, 2009-2018.

\section{Figure 5}

Spatiotemporal clusters of HFMD in mainland China, 2009-2018. 